\title{
Hider-Finder-Combiner: An Adversarial Architecture For General Speech Signal Modification
}

\author{
Jacob J Webber ${ }^{1}$, Olivier Perrotin ${ }^{2}$ and Simon King ${ }^{1}$ \\ ${ }^{1}$ The Centre for Speech Technology Research, University of Edinburgh, United Kingdom \\ ${ }^{2}$ Univ. Grenoble Alpes, CNRS, Grenoble INP, GIPSA-lab, France \\ j.j.webberded.ac.uk
}

\begin{abstract}
We introduce a prototype system for modifying an arbitrary parameter of a speech signal. Unlike signal processing approaches that require dedicated methods for different parameters, our system can - in principle - modify any control parameter that the signal can be annotated with. Our system comprises three neural networks. The 'hider' removes all information related to the control parameter, outputting a hidden embedding. The 'finder' is an adversary used to train the 'hider', attempting to detect the value of the control parameter from the hidden embedding. The 'combiner' network recombines the hidden embedding with a desired new value of the control parameter. The input and output to the system are mel-spectrograms and we employ a neural vocoder to generate the output speech waveform. As a proof of concept, we use $F_{0}$ as the control parameter. The system was evaluated in terms of control parameter accuracy and naturalness against a high quality signal processing method of $F_{0}$ modification that also works in the spectrogram domain. We also show that, with modifications only to training data, the system is capable of modifying the $1^{\text {st }}$ and $2^{\text {nd }}$ vocal tract formants, showing progress towards universal signal modification. Index Terms: speech synthesis, adversarial networks, speech modification
\end{abstract}

\section{Introduction}

Current speech synthesisers are typically [1] separated into two main components: the sequence-to-sequence model converts sequences of graphemes or phonemes into sequences of frames in the frequency domain, then a neural vocoder generates a waveform. These systems yield high quality results, but are limited to synthesis of voices for which audio exists. This is true even when using adaptation, by injecting high-level information (speaker identity [2], speaking style [3], expressivity, etc.) into the model. Whilst current systems have impressive adaptation ability [4], they are driven by data and generally offer no explicit control of speech parameters such as pitch or timbre. Some recent neural vocoders [5, 6] accept an explicit pitch parameter as input but at the expense of speech quality [7].

Using signal processing, manipulation of arbitrary speech parameters is challenging, not least because speech parameters co-vary. However, using signal processing only to extract parameters - to annotate waveforms - is far less challenging.

The limitations of current neural approaches, and the relative ease of annotating speech compared to signal processing manipulation, together motivate the approach presented here.

The first author is funded by the Engineering and Physical Sciences Research Council (grant EP/L01503X/1), EPSRC Centre for Doctoral Training in Pervasive Parallelism at the University of Edinburgh, School of Informatics.
We aim for true controllability of speech. This is performed in the mel-spectrogram domain because of its ability to represent most aspects of speech and its widespread use as the interface between sequence-to-sequence models for speech synthesis neural vocoders $[8,9]$. We present a proof of concept for a machine learning-based approach to modification of speech signals for any arbitrary control parameter, which requires only a training dataset annotated with this control parameter. The method aims to modify precisely one control parameter whilst leaving all other aspects of the speech signal unchanged.

\section{Controllability}

\subsection{What is Ideal Control?}

We can annotate many parameters on a speech signal; e.g., $F_{0}$ (perceived as pitch), spectral tilt (voice quality), or formant positions (articulation) can be estimated automatically from waveforms. We could imagine annotation of other parameters using external information (e.g., physical articulator positions) or human perception (manual labelling). Ideal controllability implies being able to modify one parameter while changing as few other parameters as possible. However, many parameters are deeply intertwined with others. For example, mel-spectrograms depend on (at least) $F_{0}$, speaker characteristics and linguistic content. Ideal control of $F_{0}$ means changing only that part of the mel-spectrogram which is dependent on $F_{0}$.

\subsection{Signal Processing for Speech Modification}

Vocoders achieve general-purpose speech parameter manipulation by decomposition of the signal into source and spectralenvelope features. For example, STRAIGHT [10] or WORLD [11] use smooth spectral envelope features [12] which allow spectral modification independently from $F_{0}$ and duration. Nevertheless, they are limited to controlling parameters which can be mapped to/from those speech features. GFMIAIF [13] represents the spectral envelope with vocal tract and glottis-related parameters, for greater controllability [14]. Nonparametric methods avoid decomposition of the signal but are generally restricted to modifying one parameter. For instance, TD-PSOLA [15] and phase vocoders [16] are capable of highquality $F_{0}$ and duration modification, but do not generalise to other control parameters. Although such methods are effective, modifying one parameter often fails to preserve its natural covariation with other parameters, resulting in artefacts.

\subsection{Controllability and Machine Learning}

Our proposed system uses machine learning techniques to modify a speech signal by an arbitrary control parameter. Conventional neural networks are learned universal function ap- 
proximators. Consider the function $f$ that maps input melspectrogram $x$ to output mel-spectrogram $z$ and approximate it with a network $F$ trained to minimise error $\epsilon=|f(x)-F(x)|$ averaged over all $(x, z)$ in some training set. If we add another control parameter $y$ as input to the network, so that $z \approx F(x, y), F$ will simply learn to ignore $y$ if $x$ contains sufficient information to predict $z$. Even if $x$ contains only partial information, when we attempt to control the speech $z$ by varying control parameter $y$ we risk contradicting information contained in $x$. To solve these related problems, we employ a hidden representation $h$ which contains as little information as possible about $y$, but all other information from $x$ that, when combined with $y$, will predict $z$. We use a hider $(H)$ and combiner $(C)$ network for this, with a finder $(D)$ adversary.

$$
\begin{aligned}
h & =H(x) \\
z & \approx C(h, y) \\
y & \approx D(h)
\end{aligned}
$$

This is similar to a Generative Adversarial Network (GAN), in which a generator network aims to fool a discriminator (adversary) network into misclassifying its output, e.g., as being genuine [17]. Generator and discriminator are trained in turn. In our system, the finder is the discriminator.

\section{The Proposed Architecture}

Fig. 1 shows the architecture, and [18] describes how this arose out of our earlier experiments:

1. The hider takes as input a mel-spectrogram and produces as output a 2-dimensional hidden embedding (one axis is time, as in the spectrogram) which preserves sufficient information for reconstructing the spectrogram but as little information about the control parameter as possible.

2. The finder network is an adversary that attempts to detect the value of the control parameter from the output of the hider. It is only used to train the system and is not required during generation.

3. The combiner network takes as input the hidden embedding and a new value of the control parameter and produces as output a mel-spectrogram.

In the following, we use $F_{0}$ as an example control parameter. The architecture is trained from scratch without any supervision of the individual networks, using only ground-truth melspectrograms paired with corresponding control parameter values; input and output are identical in training. The goals of training are (1) when given the original value of the control parameter, the combiner output is the same as the hider input, and (2) the finder cannot detect the value of the control parameter from the hider output.

Hider: Input ground-truth mel-spectrograms are passed through a fully-connected layer, into a convolutional layer of kernel size 10, then a 3-layer 800-node GRU with tanh activation. The features are resized to the correct dimensionality using a fully-connected layer.

Finder: Hidden embeddings are passed directly into a 2layer, 300-node stacked GRU with tanh activation, then one linear layer, and finally a softmax layer which produces a probability distribution across the quantised $F_{0}$ values.

Combiner: The architecture of the combiner is shown in Fig. 2. Inputs are a voicing flag, the control parameter, and the output of the hider network. The combiner first passes a

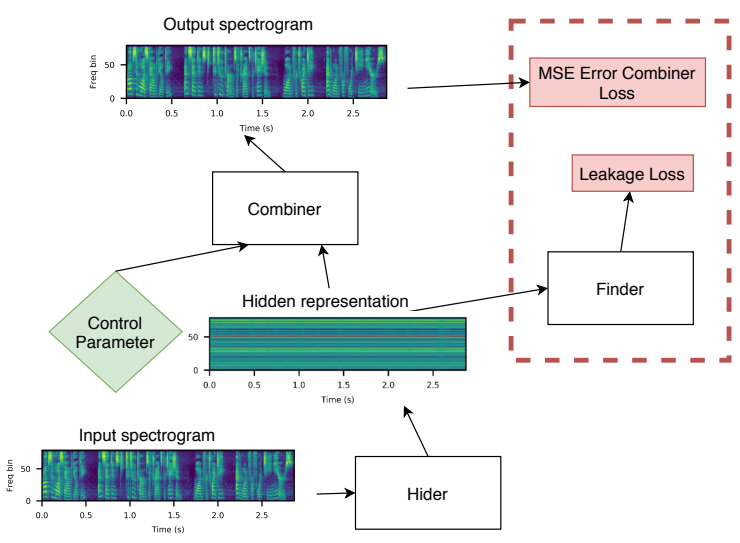

Figure 1: The complete architecture. Components in the dashed box are used only during training.

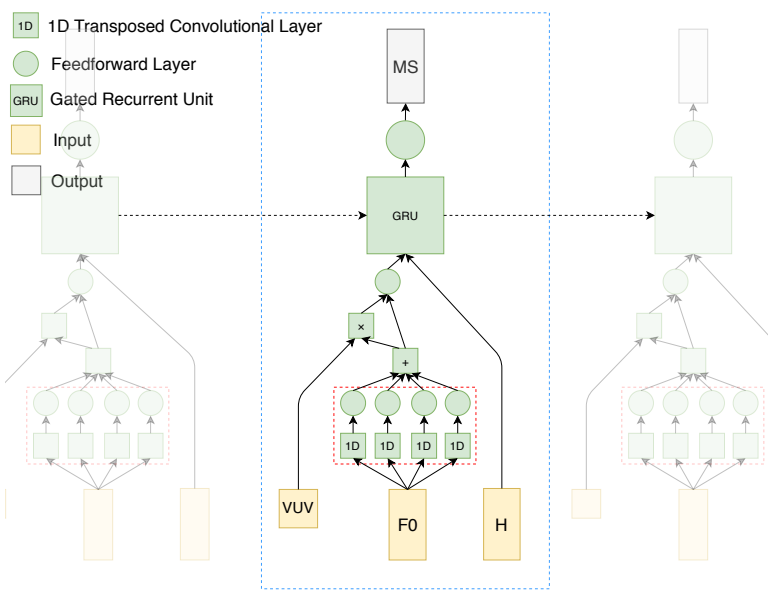

Figure 2: The combiner network architecture across three time frames. The blue dotted box shows one frame. The red dotted box shows a parallel bank of transposed convolutions (PBTC). $H$ is the output of the Hider network, VUV is a voiced/unvoiced flag, $F_{0}$ is the control parameter.

1-hot embedding of the control parameter through a parallel bank of transposed convolutions (PBTC). A PBTC consists of an array of 1D transposed convolutional layers, with each of these convolutions using a different dilation and the results being summed together. The aim of this PBTC is to generate the harmonic structure in the spectrogram characteristic of $F_{0}$. Because convolutional networks are powerful, this approach generalises to other features where a single control parameter results in structured spectral energy. The output of the PBTC is duplicated, with one of the two copies being masked by voicing (zeroed out in unvoiced regions). This duplication allows the system to learn whether to take into account voicing information, and was found to reduce artefacts in unvoiced sections of speech. For the PBTC, 10 dilated transposed convolutional layers were used with kernel size 50 and dilations in the range 2-20. The result of the PBTC is concatenated with the hidden embeddings coming from the hider, and passed to a 3-layer 1200-node stacked GRU with tanh activation. Finally, a fully-connected layer outputs a mel-spectrogram.

\subsection{End-to-end Adversarial Training}

The control parameter in initial experiments was $F_{0}$. Training data was automatically annotated for $F_{0}$ with WORLD's pitch 
tracker [11] and interpolated through unvoiced sections, then quantised into 80 linear-scale bins between $60 \mathrm{~Hz}$ and $500 \mathrm{~Hz}$. We refer to this vector as 'ground truth $F_{0}$ '. Similar to GANs, a two stage process is used to update models at each training step. First, the finder (adversary) is trained. For each utterance, the input mel-spectrogram is passed through the hider. It outputs a hidden embedding that is used to train the finder, with ground truth $F_{0}$ as target, to minimise cross-entropy loss. Second, the hider and combiner networks are jointly trained by backpropagating through both networks to minimise an adversarial loss defined as the weighted sum of the combiner loss, $\mathcal{L}_{\text {combiner }}$ and the leakage loss, $\mathcal{L}_{\text {leakage. }}$

$$
\mathcal{L}_{\text {adversarial }}=\mathcal{L}_{\text {combiner }}+\beta \cdot \mathcal{L}_{\text {leakage }}
$$

The MSE combiner loss is measured at the output of the combiner, whose target is the same as the input to the hider: a ground-truth mel-spectrogram. The combiner also takes ground-truth $F_{0}$ as an input.

The leakage loss measures how much information related to the control parameter is 'leaked' by the hider into the hidden embedding. Defining a representation that contains no information pertaining to a specific variable is non-trivial. Mutual information quantifies the amount of information that one random variable contains about another and this would be applicable to neural networks, which map from one random variable to another. However, calculating mutual information is difficult and can require a large number of training samples [19].

We used a simpler solution: the hider network should produce a hidden embedding from which the finder outputs a uniform probability distribution over the quantised control parameter (CP): it should be maximally uncertain about the $\mathrm{CP}$. The MSE error between finder output and a uniform distribution is equivalent to the variance of the distribution and thus the leakage loss was defined. A high value implies the finder network has certainty about the CP. Zero loss implies that all $\mathrm{CP}$ values are equally probable, because the hider has completely removed all information about the $\mathrm{CP}$ from the hidden embedding. Maximal uncertainty at finder output is preferable to a confident-butincorrect finder. In the latter case, the hider could learn to fool the finder network with a solution that is easier to learn than actually hiding the $\mathrm{CP}$ - perhaps, where $F_{0}$ is the $\mathrm{CP}$, by doubling $F_{0}$ via only removing odd harmonics - but that the combiner could use instead of its control parameter input value.

\subsection{Experimental Setup}

The LJSpeech corpus [20] was used for training, comprising recordings of 50 chapters of non-fiction books by a female US English speaker. The last chapter was held out for the evaluation. Batch size was 1 utterance with 12693 training steps per epoch. Parameters were updated using the Adam optimizer [21] after every training step. A validation set of 128 randomlyselected utterances was used for early stopping and all systems reported below were trained for 6 epochs. For the input and output, as in [8], a frame size of $50 \mathrm{~ms}$ and a frame shift of $12.5 \mathrm{~ms}$ are used to extract an 80-band mel-spectrogram with LibROSA [22]. A feature width of 80 was also selected for the hidden embedding. The audio sampling rate was $22.05 \mathrm{kHz}$.

The value of $\beta$ in eqn. 4 determines how much the leakage of control parameter value into the hidden embedding contributes to training. At $\beta=0$ there is no adversarial loss, the hidden embedding will contain control parameter information, and the combiner is free to ignore its input control parameter. Small non-zero values of $\beta$ lead to partially-modified speech, as

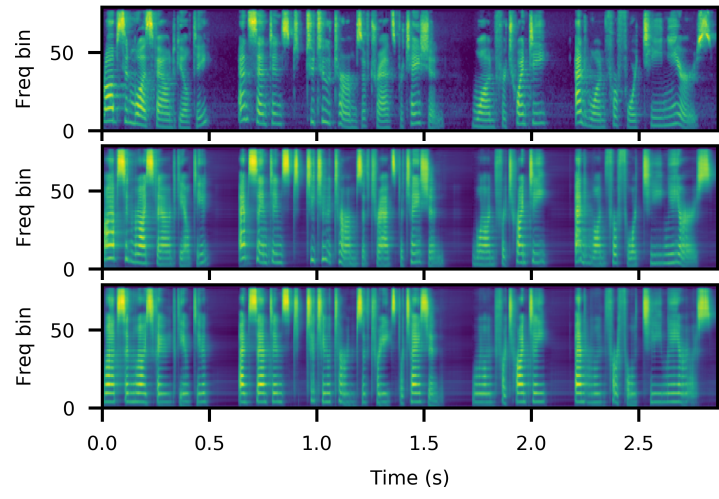

Figure 3: Example of changing $F_{0}$ to a constant value of $214 \mathrm{~Hz}$. Top: ground truth mel-spectrogram. Middle: output with $\beta=200$. Bottom: output with $\beta=800$.

shown in Fig. 3. Increasing $\beta$ brings the output speech closer to the desired control parameter value, (bottom of Fig. 3) but excessively large values of $\beta$ cause output quality to degrade because too little importance is given to the combiner MSE loss. Overall, the MSE combiner loss is positively correlated with $\beta$ and the leakage loss is negatively correlated. Pilot experiments [18] showed that $\beta=560$ is a satisfactory trade-off between output quality and control accuracy, and this value is used in the following evaluation.

\section{Evaluation}

The system was evaluated with $F_{0}$ as the control parameter (CP). We selected 30 utterances from the test chapter, and defined four control types: the original $F_{0}$ trajectory (copy); scaled $F_{0}$ by $-50 \%$ to $+50 \%$ of its original value in steps of 10 ; entirely new, but plausible, $F_{0}$ contours generated through human performance ('drawn') as in [23], with average value set to match the original $F_{0}$. As one baseline, we used a digital signal processing (DSP) approach that also operates on mel-spectrograms. All audio was generated from the baseline and the proposed Hider-Finder-Combiner approach (HFC) melspectrograms by an open-source implementation of a state-ofthe-art neural vocoder $[9,24]$. As a second baseline, we used WORLD, with speech waveforms as input and output. We provide an objective evaluation of how closely the output of our system follows the specified control parameter value, and a subjective evaluation of the audio quality.

\subsection{The Baseline DSP System}

To disentangle the harmonics of $F_{0}$ and vocal tract (VT) resonances (formants), in order to allow $F_{0}$ modification without changing formants, source-filter separation is performed with GFM-IAIF [13] on mel-spectrograms. This decomposes the spectral envelope into two sets of linear predictive (LP) coefficients [25], modeling the glottis and the VT respectively. $F_{0}$ scaling is applied to the excitation signal obtained by inverse filtering the speech frame with the glottis and VT filters. This modification follows the principle of the phase vocoder [16], modified slightly to use mel-spectrograms: since phase reconstruction is done by the neural vocoder, the DSP system only performs frequency-stretching or compressing on the amplitude spectrum by a rescaling factor. Also, each mel-spectrogram frame is interpolated to a linear frequency scale before $F_{0}$ modification, then mel-scaled again afterwards. Finally, the speech 


\begin{tabular}{|c|ccc|}
\hline & WORLD & DSP & HFC \\
\hline$F_{0}$ copy & 0.16 & 0.16 & 0.16 \\
$F_{0}$ scale & 0.18 & 0.20 & 0.23 \\
Drawn $F_{0}$ & 0.15 & 0.18 & 0.14 \\
\hline \multicolumn{4}{|c}{} \\
\hline$F_{1}$ & GFM-Voc & DSP & HFC \\
$F_{2}$ & 0.33 & 0.26 & 0.36 \\
\end{tabular}

Table 1: RMSE (in octave) of $\log _{2}\left(F_{0}\right), \log _{2}\left(F_{1}\right)$ and $\log _{2}\left(F_{2}\right)$ between control and synthesis for the models in consideration.

frame is reconstructed by filtering the $F_{0}$-scaled source signal with both glottis filter and VT filters before going back to the mel-frequency scale to feed the neural vocoder.

\subsection{Objective Evaluation}

To assess the fidelity of the output to the control parameter, we extracted $F_{0}$ from the synthesised speech waveforms with WORLD and measured the root mean square error (RMSE) with their respective $F_{0}$ control values, on a logarithmic scale. Table 1 (top) shows the median values of the $\log _{2}\left(F_{0}\right)$ RMSE distributions for each system and $F_{0}$ modification type. A Kruskal-Wallis rank-sum test using a $\chi^{2}$ distribution shows that the RMSE differences between models are not significant for copy $\left(\chi^{2}=1.2, p=0.55\right)$, are significant for scale $\left(\chi^{2}=49.3\right.$, $\left.p<1 e^{-10}\right)$ and marginally significant for drawn $F_{0}\left(\chi^{2}=7.2\right.$, $p<0.03)$. A post-hoc Dunn test for pairwise comparison between methods assesses that all measures are different for scaled and drawn $F_{0}(p<0.01)$, except between HFC and WORLD for drawn $F_{0}(p=0.5)$. The proposed system performs as well as the others for copying the $F_{0}$ trajectory. For $F_{0}$ scaling, there is significant but small difference, with an RMSE of 0.02 octave higher for HFC than the DSP method. For drawn $F_{0}$, HFC performs significantly better than DSP, and as well as WORLD. From these results, it appears that HFC is better for arbitrary modification of $F_{0}$ (drawn $F_{0}$ ) than the DSP method. The superiority of the DSP method for the narrow case of $F_{0}$ scaling is not surprising, given the simplicity of stretching melspectrograms of the glottal source signal.

\subsection{Subjective Evaluation}

To assess naturalness, a comparative mean opinion score (CMOS) evaluation was performed. Listeners were asked to compare utterances in pairs on a five point scale, corresponding to whether they thought either option sounded much more natural, slightly more natural, or whether both options sounded similarly natural. Each pair comprised the same utterance generated with DSP and HFC. From our speech material, we selected synthesis with original $F_{0}$ (copy); scalings of $\pm 20 \%$ from the original $F_{0}$, and the drawn $F_{0}$, leading to a total of 120 pairs that were randomly split into two blocks. 50 native English speaking US citizens were recruited and paid using the Prolific Academic platform. 25 listeners judged block 1, and 25 others judged block 2. An equal number of pairs was presented in each order (DSP-HFC, HFC-DSP) and the ordering of pairs was randomised per listener.

Fig. 4 shows the CMOS for the three different conditions. A Kruskal-Wallis rank-sum test followed by a Dunn test for pairwise comparison shows that the drawn distribution is significantly different from the two others $\left(p<1 e^{-15}\right)$, and that the copy and scale distributions are marginally significantly different $(p<0.03)$. This evaluation shows that HFC is as good as DSP for copy and scale, and better for the generation of new $F_{0}$

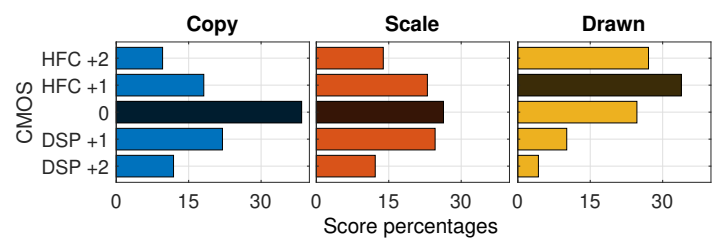

Figure 4: CMOS between DSP and HFC for different $F_{0}$ modifications. The dark bars indicate the median score for each condition.

trajectories. These results are consistent with the objective measures, and confirm that HFC is better for the most practicallyuseful case of arbitrary modification of $F_{0}$.

\section{Towards a Universal Signal Modifier}

Our approach is intended to generalise to the modification for any control parameter which can be annotated on speech waveforms. We now demonstrate this generality by modifying the first or second formant $\left(F_{1} / F_{2}\right)$, which we annotated on training data using the GFM-IAIF VT filter [13]. $F_{1}$ and $F_{2}$ are significantly more challenging to manipulate than $F_{0}$. As with $F_{0}$, the control parameter was quantised into 80 linearly spaced bins: $F_{1}$ between 0.2 and $1 \mathrm{kHz}, F_{2}$ between 0.5 and $3 \mathrm{kHz}$.

We synthesised the 50 sentences of median length from the test chapter in which we scaled either $F_{1}$ or $F_{2}$ by between $-40 \%$ and $+40 \%$ of their original value in steps of 20 . Both HFC and DSP methods were compared against GFM-Voc [14], which extracts the VT filter with GFM-IAIF, changes the formant position through pole modification, then filters the unchanged source signal to reconstruct the speech waveform. This is the same process that is applied in DSP, except that in the former, the waveform is obtained by inverse Fourier transform of the spectrogram, using the original phase, while in the latter the mel-spectrogram is fed to the neural vocoder. Since GFM-IAIF is used for both data annotation and parameter modification in DSP, we used Praat [26] for independent extraction of $F_{1}$ and $F_{2}$ values from all the syntheses, and the RMSE to their respective control trajectories was computed and is shown in Table 1 (bottom). A Kruskal-Wallis rank-sum test paired with a posthoc Dunn test shows that distributions for each method are significantly different for both $F_{1}$ and $F_{2}\left(p<1 e^{-3}\right)$. Although formant modification accuracy is lower for HFC than the other methods, the RMSE difference remains below 0.1 octave in all cases, demonstrating that HFC can modify formants using precisely the same architecture as for modifying $F_{0}$. Subjective evaluations of $F_{1}$ and $F_{2}$ modification are left as future work, but informal tests confirm the output quality is high. Audio samples from all systems described in this paper are available online $^{1}$.

\section{Conclusion}

We have presented a novel approach for explicit control of an arbitrary speech parameter and shown that the system can generate high quality and accurate output for two very different categories of control parameter, one related to the glottal source and the other the vocal tract shape. Future work includes subjective evaluation of $F_{1} / F_{2}$ modification, as well as testing the modification of new parameters to further demonstrate the universality of our approach.

\footnotetext{
${ }^{1}$ Audio samples: http://homepages.inf.ed.ac.uk/ s1116548/interspeech-2020
} 


\section{References}

[1] Z. Kons, S. Shechtman, A. Sorin, C. Rabinovitz, and R. Hoory, "High quality, lightweight and adaptable TTS using LPCNet," in Proc. of Interspeech, Graz, Austria, September 15-19 2019, pp. 176-180.

[2] R. S. Doddipatla, N. Braunschweiler, and R. Maia, "Speaker adaptation in DNN-based speech synthesis using d-vectors," in Proc. Interspeech, Stockholm, Sweden, August 2017, pp. 3404 3408 .

[3] Y. Wang, D. Stanton, Y. Zhang, R. J. Skerry-Ryan, E. Battenberg, J. Shor, Y. Xiao, Y. Jia, F. Ren, and R. A. Saurous, "Style tokens: Unsupervised style modeling, control and transfer in endto-end speech synthesis," in Proceedings of the International Conference on Machine Learning, vol. 80, Stockholmsmässan, Stockholm Sweden, July 10-15 2018, pp. 5180-5189.

[4] Y. Jia, Y. Zhang, R. Weiss, Q. Wang, J. Shen, F. Ren, P. Nguyen, R. Pang, I. L. Moreno, Y. Wu et al., "Transfer learning from speaker verification to multispeaker text-to-speech synthesis," in Advances in neural information processing systems, 2018, pp. 4480-4490.

[5] Y. Wu, T. Hayashi, P. L. Tobing, K. Kobayashi, and T. Toda, "Quasi-periodic wavenet vocoder: A pitch dependent dilated convolution model for parametric speech generation," in Proc. of Interspeech, G. Kubin and Z. Kacic, Eds., September 15-19 2019, pp. 196-200.

[6] J. Valin and J. Skoglund, "LPCNet: Improving neural speech synthesis through linear prediction," in IEEE International Conference on Acoustics, Speech and Signal Processing (ICASSP), Brighton, UK, May 2019, pp. 5891-5895.

[7] P. Govalkar, J. Fischer, F. Zalkow, and C. Dittmar, "A comparison of recent neural vocoders for speech signal reconstruction," in ISCA Speech Synthesis Workshop, Vienna, Austria, September 20-22 2019, pp. 7-12.

[8] J. Lorenzo-Trueba, T. Drugman, J. Latorre, T. Merritt, B. Putrycz, R. Barra-Chicote, A. Moinet, and V. Aggarwal, "Towards achieving robust universal neural vocoding," in Proc. of Interspeech Graz, Austria, September 2019, pp. 181-185.

[9] N. Kalchbrenner, E. Elsen, K. Simonyan, S. Noury, N. Casagrande, E. Lockhart, F. Stimberg, A. van den Oord, S. Dieleman, and K. Kavukcuoglu, "Efficient neural audio synthesis," in Proceedings of the 35th International Conference on Machine Learning, vol. 80. Stockholmsmässan, Stockholm Sweden: PMLR, 10-15 Jul 2018, pp. 2410-2419. [Online]. Available: http://proceedings.mlr.press/v80/kalchbrenner18a.html

[10] H. Banno, H. Hata, M. Morise, T. Takahashi, T. Irino, and H. Kawahara, "Implementation of realtime STRAIGHT speech manipulation system: Report on its first implementation," Acous tical Science and Technology, vol. 28, no. 3, pp. 140-146, 2007.

[11] M. Morise, F. Yokomori, and K. Ozawa, "WORLD: A vocoderbased high-quality speech synthesis system for real-time applications," IEICE Transactions on Information and Systems, vol. E99.D, no. 7, pp. 1877-1884, 2016.

[12] M. Morise, "Cheaptrick, a spectral envelope estimator for high-quality speech synthesis," Speech Communication, vol. 67, pp. $1-7,2015$. [Online]. Available: http://www.sciencedirect. com/science/article/pii/S0167639314000697

[13] O. Perrotin and I. V. McLoughlin, "A spectral glottal flow model for source-filter separation of speech," in IEEE International Conference on Acoustics, Speech, and Signal Processing (ICASSP), Brighton, UK, May 12-17 2019, pp. 7160-7164.

[14] O. Perrotin and I. McLoughlin, "Gfm-voc: A real-time voice quality modification system," in Proceedings of Interspeech, Graz, Austria, September 15-19 2019, pp. 3685-3686.

15] C. Hamon, E. Mouline, and F. Charpentier, "A diphone synthesis system based on time-domain prosodic modifications of speech,' in IEEE ICASSP, 1989, pp. 238-241 vol.1.
[16] J. L. Flanagan and R. M. Golden, "Phase vocoder," The Bell System Technical Journal, vol. 45, no. 9, pp. 1493-1509, November 1966.

[17] I. Goodfellow, J. Pouget-Abadie, M. Mirza, B. Xu, D. WardeFarley, S. Ozair, A. Courville, and Y. Bengio, "Generative adversarial nets," in Advances in Neural Information Processing Systems 27, 2014, pp. 2672-2680. [Online]. Available: http: //papers.nips.cc/paper/5423-generative-adversarial-nets.pdf

[18] J. Webber, "Controllable recurrent adversarial speech processing,' Master's thesis, The University of Edinburgh, UK, 2019.

[19] M. I. Belghazi, A. Baratin, S. Rajeshwar, S. Ozair, Y. Bengio, A. Courville, and D. Hjelm, "Mutual information neural estimation," in Proceedings of the 35th International Conference on Machine Learning, vol. 80, Stockholmsmässan, Stockholm Sweden, July 2018, pp. 531-540. [Online]. Available: http: //proceedings.mlr.press/v80/belghazi18a.html

[20] K. Ito, "The LJ Speech dataset," https://keithito.com/LJ-SpeechDataset/, 2017.

[21] D. P. Kingma and J. Ba, "Adam: A method for stochastic optimization," in 3rd International Conference on Learning Representations, ICLR 2015, San Diego, CA, USA, May 7-9, 2015, Conference Track Proceedings, 2015. [Online]. Available: http://arxiv.org/abs/1412.6980

[22] B. McFee, C. Raffel, D. Liang, D. Ellis, M. Mcvicar, E. Battenberg, and O. Nieto, "librosa: Audio and music signal analysis in python," in Proceedings of the 14th python in science conference, January 2015, pp. 18-24.

[23] M. Evrard, S. Delalez, C. d'Alessandro, and A. Rilliard, "Comparison of chironomic stylization versus statistical modeling of prosody for expressive speech synthesis," in Proceedings of Interspeech, Dresden, Germany, September 6-10 2015, pp. 33703374.

[24] Fatchord, "Wavernn implementation," 2020. [Online]. Available: http://github.com/fatchord/WaveRNN

[25] J. Makhoul, "Linear predicion: A tutorial review," Proceedings of the IEEE, vol. 63, no. 4, pp. 561-580, April 1975.

[26] P. Boersma and D. Weenink, "Praat, a system for doing phonetics by computer," Glot International, vol. 5, no. 9/10, pp. 341-347, November 2001 